\title{
Nasal sound pressure as objective verification of implant in active transcutaneous bone conduction devices
}

This article was published in the following Dove Press journal:

Medical Devices: Evidence and Research

\section{Sabine Reinfeldt' \\ Cristina Rigato' \\ Bo Håkansson' \\ Karl-Johan Fredén Jansson' \\ Måns Eeg-Olofsson ${ }^{2}$ \\ 'Department of Electrical Engineering, Chalmers University of Technology, Gothenburg, Sweden; ${ }^{2}$ Department of Otorhinolaryngology, Head and Neck Surgery, Sahlgrenska University Hospital, The Sahlgrenska Academy, University of Gothenburg, Gothenburg, Sweden}

\section{Video abstract}

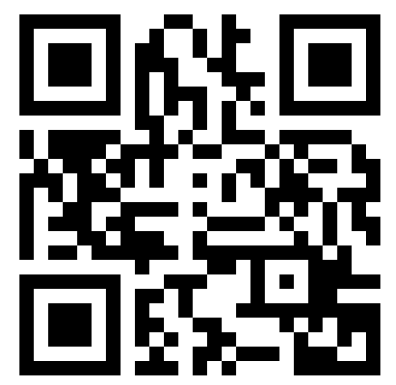

Point your SmartPhone at the code above. If you have a QR code reader the video abstract will appear. Or use: https://youtu.be/2uSR Irlaqfic
Correspondence: Sabine Reinfeldt Department of Electrical Engineering, Chalmers University of Technology,

SE-4I 296 Göteborg, Sweden

$\mathrm{Tel}+4$ 63I 7728063

Email sabine.reinfeldt@chalmers.se
Objective: Active transcutaneous bone conduction devices consist of an external audio processor and an internal implant under intact skin. During the surgical procedure, it is important to verify the functionality of the implant before the surgical wound is closed. In a clinical study with the new bone conduction implant (BCI), the functionality of the implant was tested with an electric transmission test, where the output was the nasal sound pressure (NSP) recorded in the ipsilateral nostril. The same measurement was performed in all followup visits to monitor the implant's functionality and transmission to bone over time. The objective of this study was to investigate the validity of the NSP method as a tool to objectively verify the implant's performance intraoperatively, as well as to follow-up the implant's performance over time.

Design: Thirteen patients with the BCI were included, and the NSP measurement was part of the clinical study protocol. The implant was electrically stimulated with an amplitudemodulated signal generator using a swept sine $0.1-10 \mathrm{kHz}$. The NSP was measured with a probe tube microphone in the ipsilateral nostril.

Results: The NSP during surgery was above the noise floor for most patients within the frequency interval $0.4-5 \mathrm{kHz}$, showing NSP values for expected normal transmission of a functioning implant. Inter-subject comparison showed large variability, but follow-up results showed only minor variability within each subject. Further investigation showed that the NSP was stable over time.

Conclusion: The NSP method is considered applicable to verify the implant's functionality during and after surgery. Such a method is important for implantable devices, but should be simplified and clinically adapted. Large variations between subjects were found, as well as smaller variability in intra-subject comparisons. As the NSP was found to not change significantly over time, stable transmission to bone, and implant functionality, were indicated.

Keywords: bone conduction, nasal sound pressure, bone conduction implant, ear-canal sound pressure, objective intraoperative verification

\section{Introduction}

Bone conduction devices (BCDs) are used to rehabilitate patients with conductive or mixed hearing loss. Lately, several alternatives of transcutaneous BCDs have been developed and introduced to the market. These are composed of external and internal parts with intact skin in-between. In passive transcutaneous BCDs, the internal part consists solely of one or two bone-anchored magnets in a titanium casing, while in active transcutaneous BCDs, the internal part includes a magnet 
plus a vibrating transducer, and the signal is electromagnetically transmitted through the skin, rather than mechanically transmitted. ${ }^{1}$ During implantation of these devices, it is important to check the functionality of the internal part before closing the surgical wound.

The bone conduction implant $(\mathrm{BCI})$ is a new active transcutaneous $\mathrm{BCD}$ in an ongoing multicenter clinical trial. $^{2-4}$ It consists of an external audio processor and an internal implant. The signal is electromagnetically transferred over the skin via an induction link, and the implant includes a retention magnet, a receiving coil, a demodulator, and a transducer. ${ }^{5}$ The type of transducer is a balanced electromagnetic separation transducer, to minimize the size, increase the efficiency, and decrease distortion. ${ }^{6}$ In the clinical trial, 16 patients have been included so far (13 in Gothenburg and three in Stockholm). The protocol with follow-up visits spans over a period of 5 years after fitting of the audio processor and includes several measurements, such as audiometric tone and speech tests, questionnaires, cone beam computed tomography, retention force, electric transmission, and output force level measurements.

During the surgery, a verification of the functionality of the implant is needed in order to avoid unnecessary postoperative revisions of the implant. As the patient is anesthetized during the whole surgical procedure, an objective measurement not requiring active participation by the patient is the only alternative. Furthermore, the implant needs to be stimulated in a controlled way in order to assess the quality of the response. An amplitudemodulated driver stage was developed to electrically stimulate the implanted transducer through the induction link over the skin.

In case of correct functioning of the implanted components, vibrations are transmitted to the skull bone and can therefore be recorded as vibrations in the surrounding tissue or as sound pressure in cavities of the skull. Few studies have been done on how to easily and reliably verify the transmission properties of implantable devices through laser Doppler vibrometer (LDV) and probe tube microphone measurements. In a study by Winter et $\mathrm{al}^{7}{ }^{7}$ the ear canal sound pressure (ECSP) level was found to be an effective way to verify the correct positioning and activation of the Symphonix Vibrant ${ }^{\circledR}$ Soundbridge (VSB) middle ear implant, intra- as well as postoperatively. In their study, the authors validated microphone measurements against vibrational LDV measurements of the stapes motion, finding a very high correlation, and extended the use of their method as a guideline for the fitting procedure of the VSB in follow-up visits. ${ }^{8}$ A modified version of the same technique was later developed by Schnabl et $\mathrm{al}^{9}$ and applied to the verification of mechanical coupling and functional integrity during the surgical implantation of the active transcutaneous BCD Bonebridge ${ }^{\circledR} \quad$ (Vibrant MED-EL, Innsbruck, Austria). In their measurement setup, the audio processor was acoustically stimulated with test tones and sound pressure was measured with a microphone perpendicularly taped on the skin in the middle section of the forehead. In a recent study by Ghoncheh et al, ${ }^{10}$ both LDV and microphone measurements were performed during surgery for the same transcutaneous BCD implantation. In their study, Ghoncheh et al used LDV at the implant surface and adjacent bone just after the implant fixation, while the ECSP level was measured at the opening of both ear canals after closing of the surgical wound. Despite a very high inter-patient variability, the two measurements were found to correlate well at a group level and the authors concluded that an ECSP measurement can be a reliable tool for implant verification.

Even though ECSP has been shown to have good potential as a verification tool, major limitations to the application of this method exist: 1) not all BCD patients have external ear canals, 2) during surgery, the pinna is folded over the ipsilateral ear canal, 3) the area around the ear being operated on is sterile, and 4) the contralateral ear canal is often barely accessible due to lateral head rest position of the anesthetized patient. Therefore, in this article, another cavity is addressed as an alternative to the ear canal - the nasal cavity.

In a preclinical study, nasal sound pressure (NSP) was investigated in 20 normal-hearing subjects and compared to the ear canal sound pressure. ${ }^{11}$ It was found that the NSP gave higher signal-to-noise ratio than the ear canal sound pressure from the same stimulation. Both ipsilateral and contralateral measurements were made from two stimulation positions behind the ear. It was also found that it is appropriate to measure $8 \mathrm{~mm}$ into the ipsilateral nostril, to let the patient hold their breath during the measurement, and to completely plug the ipsilateral nostril.

So far in the clinical study, the electric transmission test with NSP has been used on all 13 patients in Gothenburg. The data from the surgery and from the follow-up visits have been compiled in this study to assess whether this is a feasible way of verifying the implant's functionality, and to investigate inter- and intra-subject variations and change over time. 


\section{Aim of the study}

The aim of this study was to investigate:

(a) the potential of the NSP measurement as objective verification of the implant's functionality, in particular during surgery;

(b) the inter- and intra-subject variability in NSP values;

(c) the stability of NSP over time.

\section{Materials and methods}

\section{Subjects}

Test subjects were patients with the BCI implanted in Gothenburg between December 2012 and November 2016 in the clinical study. This clinical study was approved by the Swedish Medical Agency and Regional Ethics Committee in Gothenburg, and all patients signed informed consent prior to inclusion; hence, the study was conducted in accordance with the Declaration of Helsinki. Measurement of NSP was done as part of the protocol to verify the implant's functionality during surgery and follow-up visits for the 13 patients included in Gothenburg. Data on two follow-up visits are missing, 12 months for patient 3, and 3 months for patient 6 .

\section{Measurement setup}

The measurement setup is illustrated in Figure 1. With the $\mathrm{BCI}$ implant in place, the transmitter coil was placed over the skin aligned to the receiver coil in the implant. The inductive link was driven by using an Agilent 35670A (Keysight Technologies, Santa Rosa, CA, USA) as a fast Fourier transform analyzer and speech frequency signal generator, and an Agilent 33220A (Keysight Technologies) as a carrier frequency and amplitude modulation signal generator. Together, the transmitter inductive link, the Agilent 35670A, and the Agilent 33220A are referred to as the amplitude-modulated driver stage. With the implant fully functioning, the transducer is transmitting vibrations through the skull bone, and sound is radiated into cavities of the skull, such as the nostrils. The NSP was measured by inserting a small probe tube through an EAR Classic ear-plug (3M, Maplewood, MN, USA) in the ipsilateral nostril. The probe tube was attached to an omnidirectional microphone (EM-23346, Knowles Electronics, Itasca, IL, USA) with a battery-operated low noise linear pre-amplifier (Gennum LC506, Semtech Corp, Camarillo, CA, USA). The frequency response of the NSP was measured by the Agilent $35670 \mathrm{~A}$ in the frequency range of $0.1-10 \mathrm{kHz}$.

\section{Measurements}

The ear-plug was inserted $8 \mathrm{~mm}$ into the ipsilateral nostril. The further in, the higher the sound pressure; however, it should be at a depth which minimizes the risk of the probe tube touching internal walls of the nasal cavity. Measurements on normal-hearing subjects in a pilot

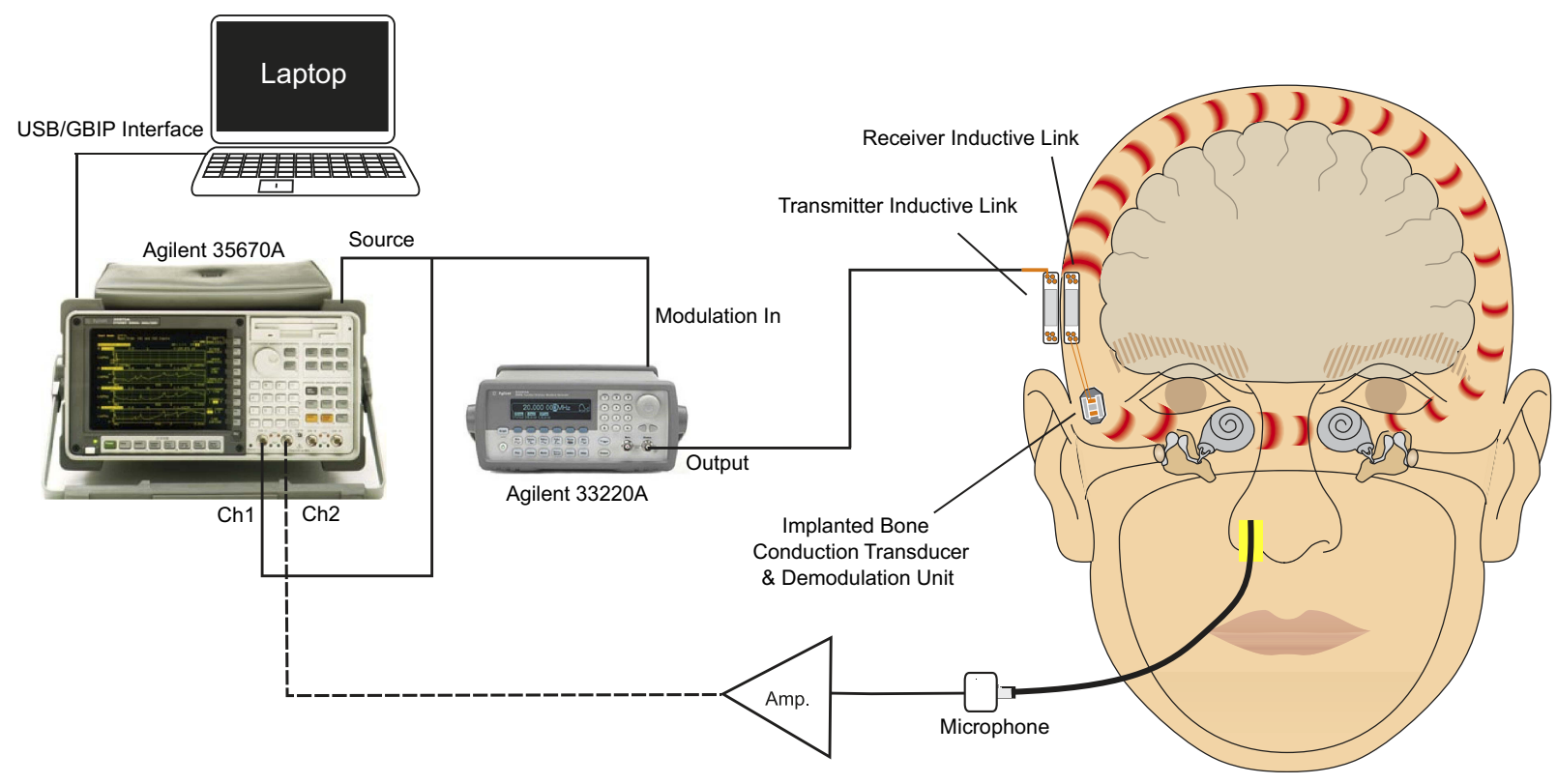

Figure I Measurement setup for the nasal sound pressure method. The amplitude-modulated driver stage, including the Agilent 35670A, the Agilent 33220A, and the transmitter inductive link, is driving the implant. The nasal sound pressure is measured by a microphone with a pre-amplifier and is analyzed by the Agilent $35670 \mathrm{~A}$. 
study ${ }^{11}$ showed that breathing gives a turbulent sound pressure response, therefore subjects were instructed not to breathe during the measurements in the follow-up visits. Given the short duration of the whole procedure (approximately 20 seconds), patients were considered able to accomplish the task of holding their breath.

\section{Calculations}

In order to generate the frequency response in $\mathrm{dB}$ rel $20 \mu \mathrm{Pa} / \mathrm{N}$ (dB SPL at $1 \mathrm{~N}$ stimulation), calibrations of the BCI transducer and the probe tube microphone were performed. The response from the transducer in the BCI was calibrated using the Skull Simulator TU-1000 (Nobelpharma, Göteborg, Sweden) to output force level. ${ }^{12}$ The probe tube microphone was calibrated using a Brüel \& Kjær (Brüel \& Kjær Sound and Vibration Measurement A/S, Nærum, Denmark) type $41341 / 2$-inch microphone. The sensitivity of the microphone was determined with a Brüel \& Kjær type 4230 sound level calibrator. Then the probe tube opening was placed $1 \mathrm{~mm}$ from the $1 / 2$-inch microphone, a sound field was introduced, and the calibration curve of the probe tube microphone was obtained for the frequency range $0.1-10 \mathrm{kHz}$.

The time analysis was based on those patients where all data were available, thus excluding two patients who missed the 3 month and the 12 month follow-up visit, respectively. The NSP data from the eleven patients used were fitted with a linear mixed effects (LME) model. ${ }^{13}$ This model was chosen over a standard linear regression model in order to consider the grouping structure of the collected data, given the fact that subsequent measurements were performed on the same patients. Observations from the same individual at different visits are expected to have a higher degree of similarity than observations from different individuals at the same visit, and the data should therefore be grouped according to the patients' ID and not only according to the time of measurement. The grouping structure would have been totally ignored in a standard regression model, where the information about individual subjects' trends is lost in the pooling process. In this study, each set of measurements taken from the same patient belongs to the same group, giving a total of eleven groups with five observations in each. By using mixed effects models, it was possible to perform a conventional linear regression, while still accounting for some extra variability associated with individual subjects. This leads to a model consisting of some fixed effects terms, describing the whole statistical population, and some random effects terms, which are associated with the individuals.
In order to reduce the between-subject variability, data points were expressed as variation from the initial value, ie, the NSP-value at fitting (baseline). The utilized model is then given by:

$$
Y_{i j}=m+k \cdot t_{i}+\beta_{j} \cdot t_{i}+\epsilon_{i}
$$

where Yij is the NSP measured at observation i for subject $\mathrm{j}$ expressed as variation from the baseline for patient $\mathrm{j}$, $\mathrm{i}=1: 5$ (measurement number), $\mathrm{j}=1: 11$ (subject ID), $\mathrm{t}_{\mathrm{i}}=0,1$, $3,6,12$ months (independent variable), $m$ is the overall intercept, $k$ is the overall slope, and $\beta_{\mathrm{j}}$ is a subject-specific parameter, which is assumed to be random, ie, for each subject the values are drawn from a normal distribution, and $\epsilon_{\mathrm{i}} \sim \mathrm{N}(0, \sigma)$ is the residual error.

With this model, it was possible to identify general trends common to all subjects by looking at the overall intercept $(m)$ and slope $(k)$. Studying the random parameter $\left(\beta_{j}\right)$ gave the possibility to identify any subject showing a trend deviating from the average.

The following assumptions were made and verified on the data in order to legitimate the use of an LME model: 1) normal distribution of the data samples, 2) independency and constant variance of the residuals (modeled value subtracted from observed data), 3) normal distribution of the residuals, and 4) normal distribution of random effects.

MATLAB (MathWorks Inc, Natick, MA, USA) was used to read and organize data in matrices, and $\mathrm{R}$ (R Core Team (2016), Vienna, Austria) was used for the statistical analysis. The model was fitted with the lme 4 package ${ }^{14}$ and the statistical significance was obtained from the $t$-values generated by the utilized lmer fitting function with $55-2=53$ degrees of freedom, where 55 is the total number of observations (eleven subjects with five observations each) and 2 is the number of fixed parameters included in the model ( $\mathrm{m}$ and $\mathrm{k}$ ). With these degrees of freedom, an estimated parameter can be defined significantly different from 0 at significance level $\alpha=5 \%(p<0.025)$ if the $t$-value is $>2.0057$.

All three model parameters were estimated for the key frequencies $0.5,1,2$, and $4 \mathrm{kHz}$, and the overall slope for every measured frequency. The estimated overall slope $(k)$ was regarded as the main indicator of transmission stability over time, where $k=0$ indicates unchanged transmission properties, while a positive or negative $\mathrm{k}$ value is associated with an increased or decreased transmission, respectively.

\section{Results}

Figure 2 shows an example of a patient's NSP for surgery and all follow-up visits. The results are shown only for the 
frequency interval $0.4-5 \mathrm{kHz}$, even though the measurements were made for $0.1-10 \mathrm{kHz}$, because this is the approximate frequency interval where the NSP is clearly above the microphone noise floor for most patients. The results illustrated that the intra-subject variability is relatively small when comparing follow-up visit measurements, where the measurement conditions are similar. At surgery, the NSP seems to show a systematically different frequency dependence, which could be attributed to the difference in measurement conditions, as during surgery the patient is lying down anaesthetized with a respiration tube down the trachea, while in follow-up visits he/she is sitting and holding their breath.

In Figure 3, averages of follow-up NSPs for all patients were plotted together with the group average, where a large inter-subject variability can be seen.

The box plot in Figure 4 shows the distribution of data measured at surgery for frequencies $0.5,1,2$, and $4 \mathrm{kHz}$. The figure shows maximum and minimum values, 25 th and 75 th

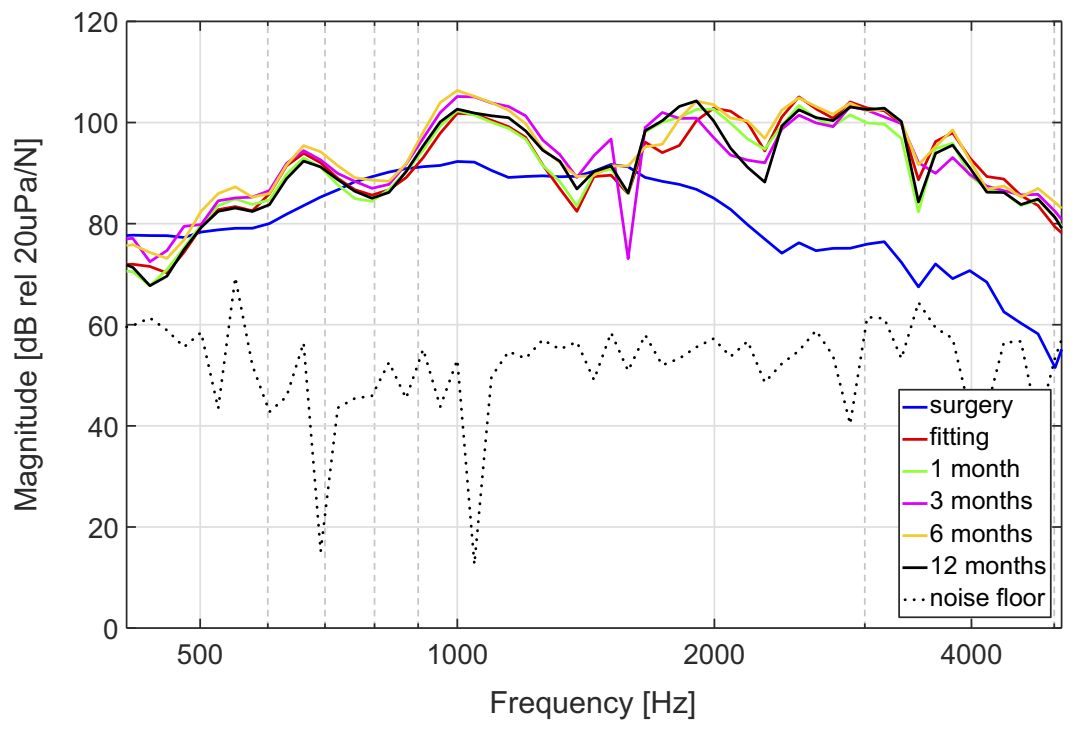

Figure 2 The nasal sound pressure for patient 12 at surgery, fitting, and follow-up visits at I, 3, 6, and 12 months after fitting. The noise floor from surgery is also presented.

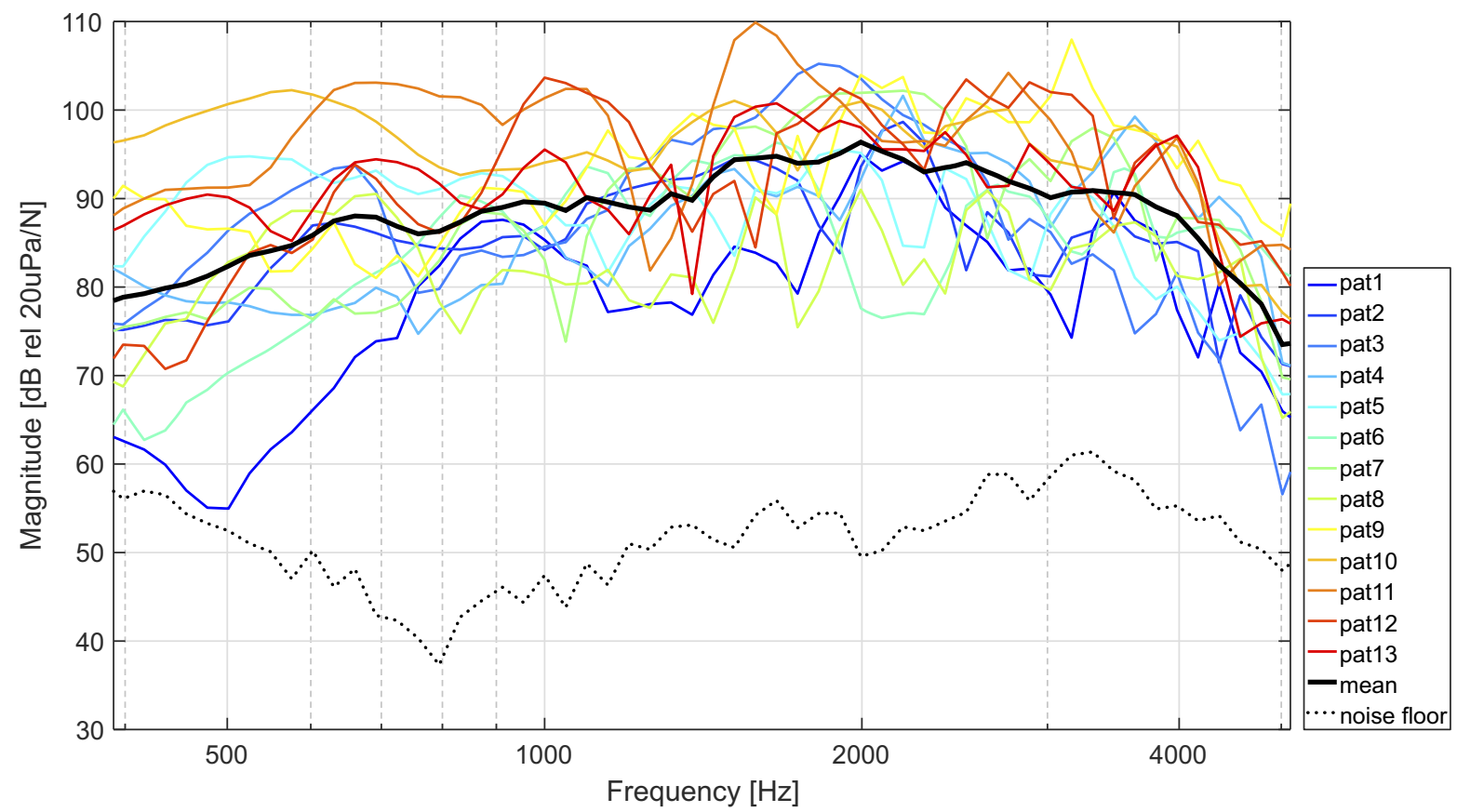

Figure 3 Average nasal sound pressure level based on follow-up data for each patient (in colors) together with the overall mean (black solid line) and the average noise floor (black dotted line). 


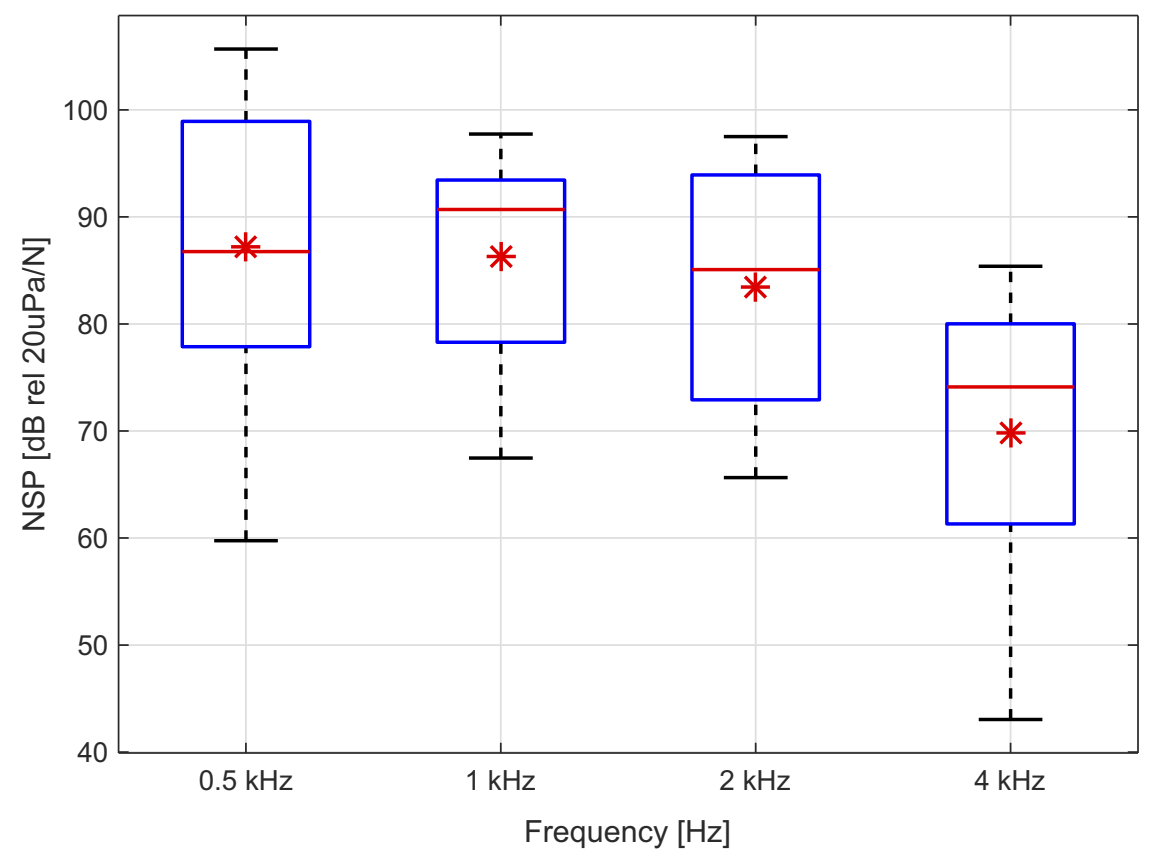

Figure 4 Boxplot of the nasal sound pressure of all patients at surgery at frequencies $0.5,1.0,2.0$, and $4.0 \mathrm{kHz}$, showing average $(*)$, median (red line), 25 and 75 percentiles (blue box), and minimum and maximum values (whiskers).

percentiles, average and median values. This representation was provided in order to get an estimate of the expected value when a new patient is measured at surgery.

The analysis of NSP data variability over time with the LME model at $500 \mathrm{~Hz}, 1,2$, and $4 \mathrm{kHz}$ is summarized in Table 1 and shown in Figures 5 and 6. Overall intercept $(m)$ quantifies the variation in baseline values, overall slope $(k)$ is associated with stability over time, and random effect is the subject-specific parameter. Subject-specific slopes represent individual variations from the average slope $k$, they are modeled as normally distributed random variables, each with a certain mean, resulting in eleven different values (one for each subject) that would superimpose to the overall $k$ to describe each subject singularly. t-values $>2.0057$ indicate a significant deviation from 0 at $5 \%$ confidence level: a condition that is not satisfied for any of the estimated parameters. Figure 5 shows the data points and their linear interpolation with a general slope and intercept (the fixed effects of the LME model). Figure 6 is a so-called caterpillar plot of the subject-specific slopes estimates (the random effect of the LME model). This figure, side-by-side, plots the eleven parameters (one for each subject) with their $95 \%$ CIs in order to facilitate the visualization and comparison of subject-specific trends. Those whose CI does not include 0

Table I Summary of results from model fitting. For the selected frequencies, a summary of the estimated fixed effects with their standard error for the linear mixed effects model is given. t-values $>2.0057$ indicate a significant deviation from $0(\alpha=5 \%)$

\begin{tabular}{|l|l|l|l|l|}
\hline & & Estimate & Standard error & t-value \\
\hline \multirow{2}{*}{$500 \mathrm{~Hz}$} & Overall intercept $(\mathrm{m})$ & 1.005 & 1.010 & 0.995 \\
\cline { 2 - 5 } & Overall slope $(\mathrm{k})$ & -0.008 & 0.184 & -0.044 \\
\hline \multirow{2}{*}{$\mathrm{f,000 \textrm {Hz }}$} & Overall intercept $(\mathrm{m})$ & 1.807 & 1.017 & 1.777 \\
\cline { 2 - 5 } & Overall slope $(\mathrm{k})$ & 0.010 & 0.225 & 0.045 \\
\hline \multirow{2}{*}{$2,000 \mathrm{~Hz}$} & Overall intercept $(\mathrm{m})$ & -0.274 & 0.655 & -0.419 \\
\cline { 2 - 5 } & Overall slope $(\mathrm{k})$ & -0.027 & 0.146 & -0.182 \\
\hline \multirow{2}{*}{$4,000 \mathrm{~Hz}$} & Overall intercept $(\mathrm{m})$ & 1.010 & 1.219 & 0.828 \\
\cline { 2 - 5 } & Overall slope $(\mathrm{k})$ & 0.467 & 0.286 & 1.634 \\
\hline
\end{tabular}



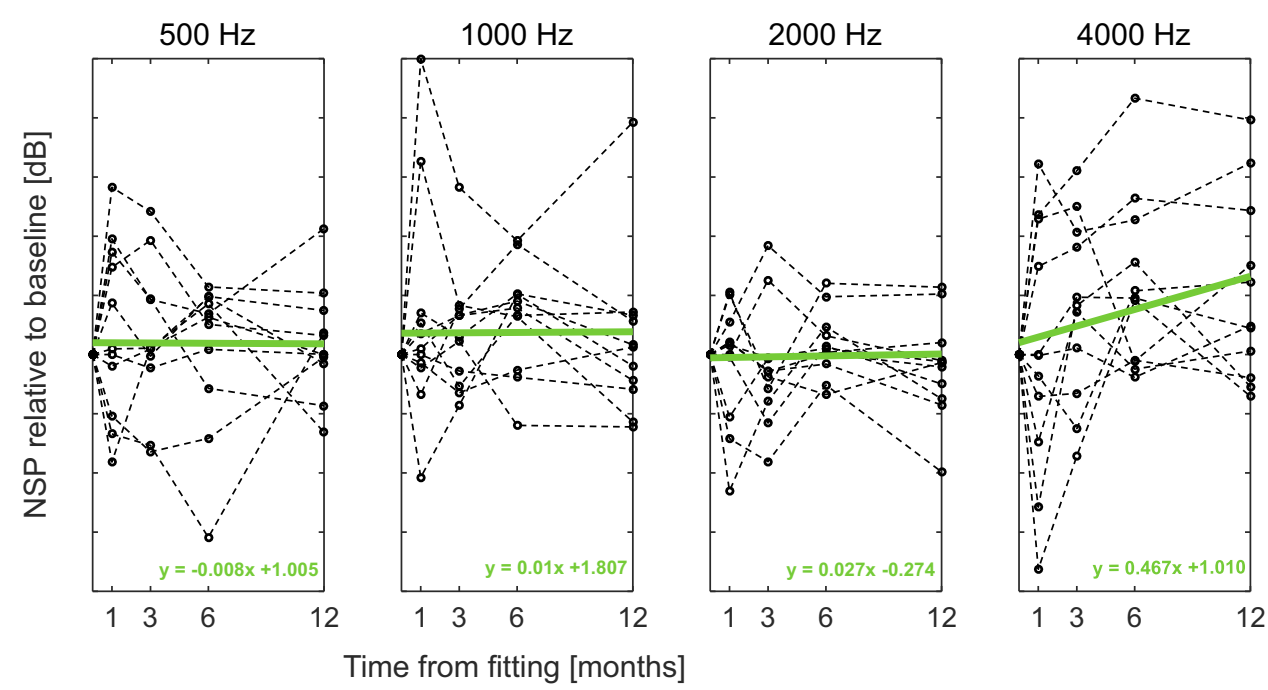

Figure 5 Linear model of the nasal sound pressure over time relative to baseline (fitting) at four frequencies with the respective equation showing slope and intercept.
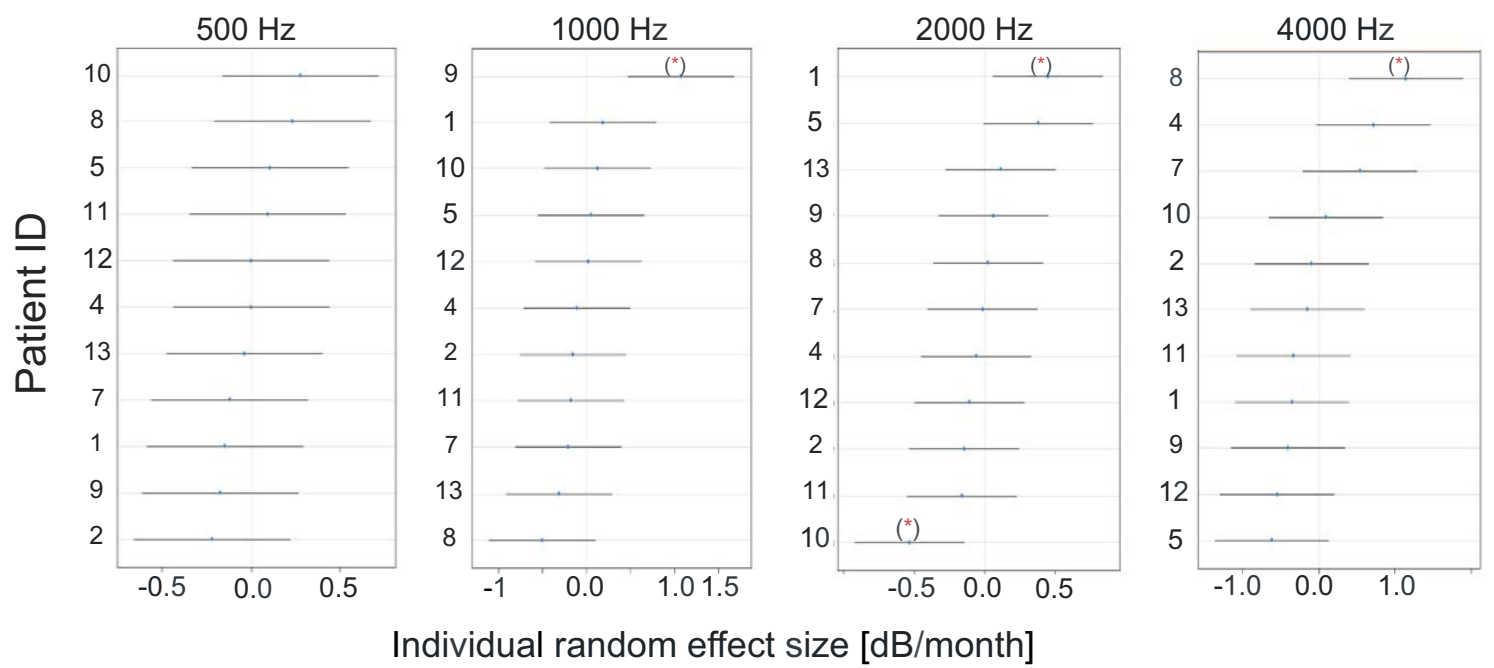

Individual random effect size [dB/month]

Figure 6 Random effects visualization: for each patient included in the analysis, the individual k-value is estimated with its $95 \% \mathrm{Cl}$. Intervals not including 0 indicate that the patient has a significant deviation from the overall slope (group average), marked with a red asterisk.

are considered as significantly deviating from the group average at 5\% confidence level.

The overall slope estimates ( $k$ values) are around zero, indicating stable transmission over time, with an exception for the value at $4 \mathrm{kHz}$, which is slightly higher. However, a further analysis of $k$ over all the frequencies (Figure 7) shows that this is the absolute highest peak value of the whole frequency interval.

\section{Discussion}

In summary, the analysis in this study has shown: 1) that NSP could be used to verify the implant's functionality during surgery, 2) large inter-subject and small intra- subject variations, 3) stability of NSP over time (12 months), and 4) difference between various measurement conditions (surgery vs follow-up).

The stability of NSP over time indicates that the transmission to bone has not changed over time. In a few frequencies, the transmission could even be interpreted as giving a slight increase in transmission (eg, $0.47 \mathrm{~dB}$ per month at $4 \mathrm{kHz}$ ), which in turn could be interpreted as osseointegration.

In the time analysis, the authors chose to include only patients where data from all follow-up visits were available. An alternative (also including the data from the two remaining patients) would have been to interpolate their missing values, or to estimate slopes ignoring the missing 


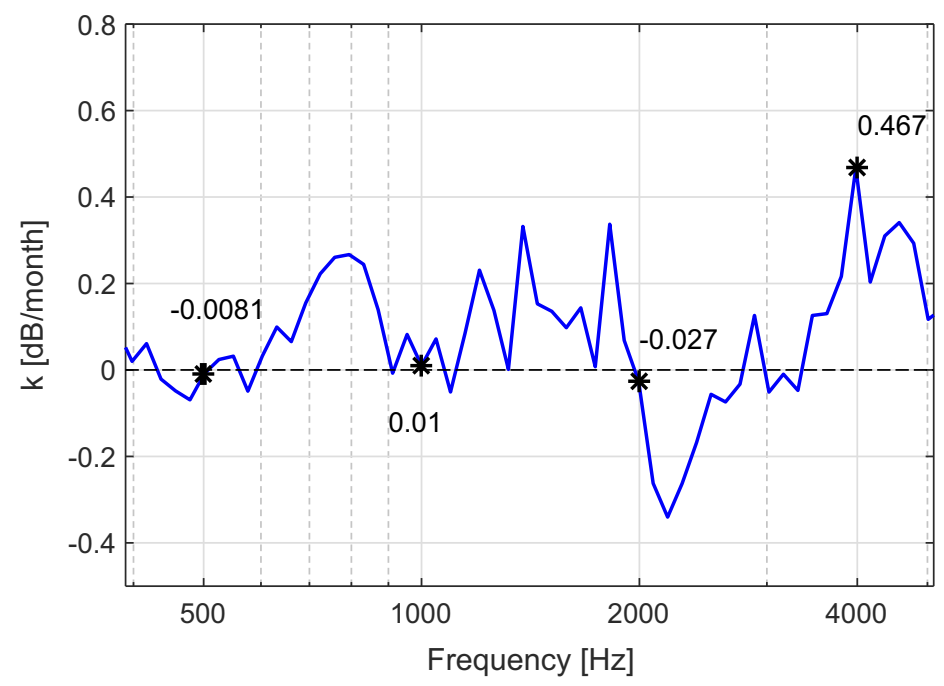

Figure 7 Slope value $(k)$ as a function of frequency. Asterisks $\left(^{*}\right)$ mark the values at the audiometric frequencies.

time points. In a comparison with the analysis performed in this article, interpolation to fill in the missing 3 month and 6 month data was done with the result that the $k$ value was very similar with no statistically significant differences from the presented results.

The decision to use a first order linear model was made mainly to keep the model as simple as possible. A second order linear model was fitted for comparison purpose. Adding a quadratic term $\left(t_{i}^{2}\right)$ to the first order formula, the following model was obtained:

$$
Y_{i j}=m+k \cdot t_{i}+k_{2} * t_{i}^{2}+\beta_{j} \cdot t_{i}+\epsilon_{i}
$$

where $k_{2}$ is the overall quadratic term coefficient at time $\mathrm{t}_{\mathrm{i}}$, and the other parameters are as defined in the data analysis section. Fitting the data with this second order model resulted in two drawbacks: 1) the complexity of the model increased, with one extra parameter to be estimated $\left(k_{2}\right)$, resulting in no apparent benefit in the quality of the fit when evaluated with the Akaike Information Criterion (AIC). The AIC by Akaike $^{15}$ is an estimator used to compare the adequacy of two or more models relative to each other, and is often used to choose between different options to fit a specific set of data; 2) the second order model for all frequencies besides $500 \mathrm{~Hz}$ showed a downwards parabolic shape, giving the impression of a decline in NSP after 6 months. This behavior is due to the fact that the data point at 12 months follow-up was lower than the one at 6 months follow-up. However, the authors believe that such an interpretation would be misleading, and hypothesize that the slight decrease of the NSP value at 12 months follow-up indicates the NSP reaching a plateau. An analysis of data for those patients who had their device for more than 36 months confirms this hypothesis. However, data from a longer time span needs to be collected before being able to estimate the long-term behavior of NSP.

One aim of this study was to investigate whether this method is suitable as objective verification of the implant during surgery and during follow-up visits. Based on our experience with these measurements, the results provided valid and reliable measurement of the implant's functionality and the transmission was stable over time. However, this measurement technique requires some technical setup, possibly too much for a standard clinical setup. On the other hand, some clinics also performing middle-ear implantation would already be equipped with such instrumentation, given that they use insert microphones in their patients' ears or on the forehead to guide the surgery and to optimize the implant coupling, according to Schnabl et $\mathrm{al}^{9}$ and Ghoncheh et al. ${ }^{10}$ Lately, a new, so-called, surface microphone approach has been developed with the objective of verifying fitting of BCDs. ${ }^{16}$ This approach has some advantages over the nasal sound probe, since it does not require the patients to hold their breath during the measurement and is easier to apply to patients.

As a subjective evaluation, the sound from radiation into the surrounding air could be listened to, but for an objective evaluation, the NSP method has been shown to be a reliable alternative. In an attempt to estimate the NSP for new patients during surgery, Figure 4 shows the distribution of NSP at a few specific frequencies. To conclude, most values above the noise floor indicate a functioning implant. Hence, 
to verify the implant's functionality, a simpler solution should be enough. However, to be able to follow the transmission to bone and the implant's functionality over time, measuring the NSP from electric stimulation appears to be an appropriate method.

As can be seen in Figure 2, the NSP during surgery in general varies from the follow-up NSP. This is hypothesized to depend on the measurement situation. To investigate this, pilot NSP measurements were made on normal-hearing subjects in various situations: in sitting and laying positions, directing the face straight ahead and to the side, and holding the breath in different ways (with open and closed mouth, and by closing the trachea with the larynx or by closing the soft palate between nasopharynx and oropharynx). All these ways were used in an attempt to estimate the difference between the surgery setting, where the patient is anesthetized with a tube in the trachea, and the follow-up measurements, where the patient is sitting, holding their breath. However, no trends showing similarity to the surgery vs follow-up difference has been found in these pilot measurements. Hence, the authors are not able to explain this difference, except that it is somehow related to the measurement conditions.

Another application of the NSP method may be to verify the functionality after accidents, ie, if the implant has been exposed to external forces, or after performing magnetic resonance imaging (MRI). Even though the implant of the BCI should withstand MRI up to 1.5 Tesla, in this clinical study it had to be removed prior to MRI, since more testing against American Standard for Testing Materials standards is required for final approval. ${ }^{17}$

In the literature, there are rare findings of studies where NSP is investigated and there are no data to compare the results of this study with. The two main application areas were judgements of nasality and measurement of noise from ventilation in intensive care. In judgements of nasality, oral and NSP levels were measured and the ratios used as a measure of nasality, as first suggested by Shelton et al. ${ }^{18}$ To investigate noise from different ventilation equipment in neonates in intensive care, sound pressure was measured in the entrance of the nostrils and further down in the postnasal space by Surenthiran et al. ${ }^{19}$ In both applications, probe tube microphones were used, and apparently without plugging the nostril, though plugging was performed in this study. The reason for plugging the nostril in this study was to reduce the influence of surrounding noise, thereby accomplishing more reliable and repeatable results.

A restriction in the results is that the noise floor of the microphone keeps the NSP valid only for a limited frequency range of $0.4-5 \mathrm{kHz}$. Therefore, the lowest and highest frequencies of the measurements have been left out in this paper. However, the authors do not consider this a major limitation, because the mid-frequency range is, in practice, the most important one that is picked-up by hearing aids.

\section{Conclusion}

The NSP under electrical stimulation of the implant was investigated in 13 patients with BCI in a clinical study. The NSP method was found suitable as objective verification of the BCI implant. It was concluded that the NSP is individualized with a large variability between patients, but also low variability within subjects during follow-up measurements. A difference was found between the NSP measured at surgery and at follow-up visits, probably due to different measurement conditions. The NSP, and hence the transmission to bone and implant functionality, were found to be stable over time.

\section{Acknowledgments}

The authors would like to thank the Swedish Hearing Research Foundation, The Swedish Research Council (Vetenskapsrådet), Vinnova and Promobilia for financial support. The study was supported by a grant from Hörselforskningsfonden (Hearing Research Foundation).

\section{Disclosure}

$\mathrm{BH}, \mathrm{SR}$, and MEO have been part-time consultants for Oticon Medical during some of the clinical study. BH has various patent IP issued. The authors report no other conflicts of interest in this work.

\section{References}

1. Reinfeldt S, Håkansson B, Taghavi H, Eeg-Olofsson M. New developments in bone-conduction hearing implants: a review. Med Devices (Auckl). 2015;8:79-93.

2. Eeg-Olofsson $\mathrm{M}$, Håkansson $\mathrm{B}$, Reinfeldt $\mathrm{S}$, et al. The bone conduction implant-first implantation, surgical and audiologic aspects. Otol Neurotol. 2014;35:679-685. doi:10.1097/MAO.0000000000000203

3. Håkansson B, Reinfeldt S, Eeg-Olofsson $\mathrm{M}$, et al. A novel bone conduction implant (BCI): engineering aspects and pre-clinical studies. Int J Audiol. 2010;49:203-215. doi:10.3109/ 14992020903264462

4. Reinfeldt S, Håkansson B, Taghavi H, Freden Jansson KJ, EegOlofsson M. The bone conduction implant: clinical results of the first six patients. Int $J$ Audiol. 2015;54:408-416. doi:10.3109/ 14992027.2014.996826

5. Taghavi H, Håkansson $\mathrm{B}$, Reinfeldt $\mathrm{S}$, et al. Technical design of a new bone conduction implant (BCI) system. Int $J$ Audiol. 2015;54:736-744. doi:10.3109/14992027.2015.1051665

6. Håkansson B. The balanced electromagnetic separation transducer a new bone conduction transducer. J Acoust Soc Am. 2003;113 (2):818-825. 
7. Winter M, Weber BP, Lenarz T. Measurement method for the assessment of transmission properties of implantable hearing aids. Biomed Tech (Berl). 2002;47(Suppl 1 Pt 2):726-727.

8. Winter M, Weber BP, Lenarz T. The use of reverse transfer function (RTF) in the fitting procedure of implantable hearing devices. Cochlear Implants Int. 2005;6:59-62. doi:10.1179/cim.2005.6. Supplement-1.59

9. Schnabl J, Wolf-Magele A, Pok SM, et al. Intraoperative measurement for a new transcutaneous bone conduction hearing implant. Otol Neurotol. 2014;35:1242-1247. doi:10.1097/MAO.0000000000000 351

10. Ghoncheh M, Lilli G, Lenarz T, Maier H. Outer ear canal sound pressure and bone vibration measurement in SSD and CHL patients using a transcutaneous bone conduction instrument. Hear Res. 2016;340:161-168. doi:10.1016/j.heares.2015.12.019

11. Shirinkar M, Ghoncheh M. Development of an Intraoperative Evaluation Method for a Novel Bone Conduction Implant Using Nasal Sound Pressure. Gothenburg, Sweden: Chalmers University of Technology; 2013.

12. Håkansson B, Carlsson P. Skull simulator for direct bone conduction hearing devices. Scand Audiol. 1989;18:91-98.
13. Liu X. Methods and Applications of Longitudinal Data Analysis. Chapter 3. Academic Press; 2016. ISBN 978-0-12-801342-7.

14. Bates D, Mächler M, Bolker B, Walker S. Fitting linear mixed-effects models using lme4. J Stat Softw. 2015;67(1):1-48. doi:10.18637/jss. v067.i01

15. Akaike H. A new look at the statistical model identification. IEEE Trans Automat Contr. 1974;19:716-723. doi:10.1109/TAC.1974.1100705

16. Hodgetts EW, Scott D, Maas P, Westover L. Development of a novel bone conduction verification tool using a surface microphone: validation with percutaneous bone conduction users. Ear Hear. 2018;39 (6):1157-1164. doi:10.1097/AUD.0000000000000572

17. Fredén Jansson KJ, Rigato C, Håkansson B, Reinfeldt S, EegOlofsson M. Magnetic resonance imaging investigation of the bone conduction implant - a pilot study at 1.5 Tesla. Med Dev Evid Res. 2015;8:413-423. doi:10.2147/MDER.S90704

18. Shelton JRL, Knox AW, Arndt JWB, Elbert M. The relationship between nasality score values and oral and nasal sound pressure level. J Speech Lang Hear Res. 1967;10:549-557. doi:10.1044/jshr.1003.549

19. Surenthiran SS, Wilbraham K, May J, Chant T, Emmerson AJB, Newton VE. Noise levels within the ear and post-nasal space in neonates in intensive care. Arch Dis Child Fetal Neonatal Ed. 2003;88:F315-F318.
Medical Devices: Evidence and Research

\section{Publish your work in this journal}

Medical Devices: Evidence and Research is an international, peerreviewed, open access journal that focuses on the evidence, technology, research, and expert opinion supporting the use and application of medical devices in the diagnosis, monitoring, treatment and management of clinical conditions and physiological processes. The identification of novel devices and optimal use of existing devices

\section{Dovepress}

which will lead to improved clinical outcomes and more effective patient management and safety is a key feature of the journal. The manuscript management system is completely online and includes a very quick and fair peer-review system. Visit http:// www.dovepress.com/testimonials.php to read real quotes from published authors. 